\title{
Urgences
}

\section{Jeux à la frontière}

\section{Oleg Stanek}

Numéro 21, novembre 1988

Bagatelles et crases

URI : https://id.erudit.org/iderudit/025497ar

DOI : https://doi.org/10.7202/025497ar

Aller au sommaire du numéro

Éditeur(s)

Urgences

ISSN

0226-9554 (imprimé)

1927-3924 (numérique)

Découvrir la revue

Citer ce document

Stanek, O. (1988). Jeux à la frontière. Urgences, (21), 91-104.

https://doi.org/10.7202/025497ar

Ce document est protégé par la loi sur le droit d'auteur. L'utilisation des services d'Érudit (y compris la reproduction) est assujettie à sa politique d'utilisation que vous pouvez consulter en ligne.

https://apropos.erudit.org/fr/usagers/politique-dutilisation/
Cet article est diffusé et préservé par Érudit.

Érudit est un consortium interuniversitaire sans but lucratif composé de l’Université de Montréal, l'Université Laval et l'Université du Québec à Montréal. Il a pour mission la promotion et la valorisation de la recherche. https://www.erudit.org/fr/ 


\section{OLEG STANEK \\ Jeux à la frontière}

Parler du «métissage», c'est évoquer le versant obscur de notre rapport à l'autre. Comme la «mésalliance» - son synonyme social -, ce terme puise sa vigueur dans le même terreau que l'imaginaire racial en général. Vouloir coiffer les différences sociales et culturelles d'un déterminisme biologique, offense aujourd'hui les règles de la bienséance; de nouvelles figures, plus acceptables, des mêmes fantasmes, viennent prendre la relève: les notions de l'«ethnie» ou de la «culture» comblent ainsi le vide laissé par celle de la «race». «Bien sûr», dirons-nous, «ils ont une âme... mais leur culture est tellement différente de la nôtre». L'identité individuelle ou collective n'a plus à craindre les mélanges du sang, mais la contagion par des substances autrement subtiles et étranges.(quoique... la psychose du SIDA ne nous rappelle-t-elle pas comment facilement on glisse d'un niveau à l'autre?). La signification du «métissage» s'est ainsi déplacée du niveau biologique-individuel à celui culturel-collectif. Si l'extérieur est source d'angoisse pour l'identité du groupe, engendrant de nouveaux racismes «culturels», un repli excessif sur soi-même ne risque-t-il pas de provoquer la stagnation, voire la dégénérescence? Comme dans le modèle bio-génétique, le «métissage» devient, à un certain degré, souhaitable, et, sous la forme d'union eugénique - comme cure de rajeunissement -, il acquiert le droit de cité. Il est entendu toutefois, que ses conséquences doivent être maîtrisées et que l'apport de l'autre puisse être digéré et incorporé à la substance intangible du groupe.

Repenser le monde social comme doublure du monde naturel celui des substances ou des combinaisons génétiques - semble être le fondement imaginaire des fantasmes du métissage. A un pôle se tapit l'angoisse de perdre l'objet du désir (par sa flétrissure ou par la corruption des qualités permettant son accès); à l'autre point l'espoir de sa reconquête, de sa reconstitution à partir des fragments épars, par une alchimie purificatrice. Le «métissage» renvoie ainsi au domaine de la production désirante, affairée à travailler les substances imaginaires: tantôt elle s'évertue à préserver leur pureté - en les protégeant de la corruption -, tantôt elle les mélange, à la recherche des formes parfaites et des éléments nobles. Même les variantes les plus conservatrices de l'imaginaire racial laissent transparaître cette fonction productive: dominées par une crainte absolue du contact avec l'autre, elles sont intarissables lorsque vient le moment de dépeindre ses conséquences maléfiques. 
Il s'agit donc de comprendre pourquoi la représentation des rapports sociaux (et leur substrat symbolique) emprunte si volontiers l'apparence de relations entre les substances, de leur confusion ou de leur séparation. Mais ce problème en comporte deux autres: quel est le mode de production de cet univers fantasmatique et qui le contrôle? $\mathrm{Ne}$ s'agit-il pas d'un simple camouflage des rapports de domination et de l'occultation des intérêts véritables des classes? Il y a de ça, sans doute. Mais une telle réponse est doublement insuffisante: d'abord parce qu'elle se borne à suggérer une finalité sans rendre compte de la forme permettant de l'atteindre. Ensuite, parce que l'univers enchanté d'où jaillit le sens du terme «métissage», ne semble pas être une addition illusoire aux rapports sociaux, mais plutôt leur élément constitutif, assurant leur cohésion et leur efficacité: c'est ainsi que la dissolution du corps miraculé du Prince, comme incarnation de la structure imaginaire du corps social, fait apparaître soudainement les rapports sociaux sous le jour cru de rapports de classe, et sonne le glas de l'Ancien régime. Foucault ${ }^{1}$ montre bien ce déplacement par l'exemple du supplice: tant et aussi longtemps que le Roi demeure investi du pouvoir de défendre le cercle magique protégeant la cité contre les forces démoniaques extérieures, le supplice conserve sa fonction cathartique. L'acharnement sur le corps du coupable réalise rituellement les conséquences de la faute, et colmate ainsi la brèche ouverte dans l'armure de la cité, par où les forces du mal pourraient s'engouffrer et submerger la société tout entière. Le supplice est alors l'occasion de réaffirmer le lien social et de restaurer l'ordre menacé. Mais ses effets seront renversés par l'effondrement de leurs assises symboliques: désormais il apparaîtra comme déchaînement de la violence de classe, représentée par le Roi, contre le peuple s'identifiant au supplicié. Ce qui a changé, ce n'est point la machine punitive, mais le fondement imaginaire du lien social.

Prendre le «métissage» pour indice d'une problématique plus vaste, nous convie à poser de multiples questions: comment sont produites les «substances» définissant les identités et les appartenances, quelle type de pouvoir garantit l'efficacité imaginaire et symbolique de l'ordre social? En quoi consiste ce noyau, en apparence solide, éprouvé comme nature de l'individu, des groupes, voire des choses, et garant de leur identité? Se compose-t-il d'une matière germinale (hérédité, âme, alluvions de 1'Histoire...?) portant en puissance ce que nous sommes ou imaginons être ou, au contraire, notre nature n'est-elle qu'un décalque d'un ordre externe, déterminant les conditions générales de notre exis- 
tence? Et si la dernière solution est la bonne, en quoi réside l'expérience fondatrice d'une telle «condition humaine»? Est-elle immédiatement sociale, ou résume-t-elle la contrainte subie par l'animal humain lorsqu'il se heurte au monde de l'Autre?

Pour éluder ces questions - est-il possible de faire autrement? sans pour autant évacuer la totalité du problème, nous intercalerons entre la Nature et la Société un troisième niveau que nous appellerons symbolique. Le terme doit être entendu au sens large: la capacité d'opérer des substitutions dans les séquences productives. Sa genèse renvoie au mythe des origines, dont d'ailleurs notre propos n'est probablement qu'une variante. Nous postulons, au départ, l'existence de processus productifs élémentaires, dans lesquels la jouissance et la souffrance sont consommées directement, sans aucune médiation. Pour construire le sujet, il faut briser cette confusion de l'immédiat; ceci peut être accompli de deux manières différentes: soit en soustrayant un élément essentiel au déroulement du processus, soit en y introduisant un corps étranger. Le sujet du processus - il n'est encore presque rien, à peine une surface d'enregistrement des effets et l'instance définissant la finalité - peut réagir de deux façons: d'abord, il peut s'efforcer de reconstituer son activité originelle en introduisant une nouvelle séquence, visant à remplacer (ou à détruire) l'élément manquant (ou le corps étranger). Mais il peut aussi tenter de le récupérer (ou de le faire disparaître) en recourant à une stratégie plus complexe: la responsabilité de la rupture étant attribuée à l'Autre (lieu d'où émane l'obstacle ou dépositaire de l'élément manquant), le sujet peut offrir à l'Autre un équivalent (en se plaçant donc en position de répondre au désir de l'Autre). Nous appellerons objet $d u$ désir le signe dont le representamen est la rupture du processus élémentaire. Dans tous les cas, son apparition produit une analyse de la production, la scindant en deux segments complémentaires: le premier, chargé de dimensions pénibles et du renoncement, s'épuise dans la production du substitut de l'objet; le second, repoussé parfois indéfiniment, concentre toute la jouissance: celle-ci n'est plus éprouvée comme exercice de la capacité productive du sujet, mais semble sourdre de l'objet lui-même. Selon le champ de l'activité productive, le premier segment peut être appelé tantôt travail, tantôt quête initiatique; le second, tantôt consommation, tantôt bonheur. La rupture de l'unité originelle traduit le passage de l'état de Nature à celui de Culture; dans l'économie désirante, elle est synonyme de l'interdiction de l'inceste et de l'affirmation radicale de l'objectalité du désir. 
Le sujet est d'abord uniquement défini à l'intérieur du processus productif: comme instance lui imposant une finalité et surface d'enregistrement de ses effets. Lorsque l'unité de ce processus est brisée, il apparaît certes un rapport à l'objet, mais étant réalisé dans la production, ce rapport demeure irréfléchi (c'est une secondéité au sens de Pierce). Il faut donc une nouvelle surface susceptible de représenter la place du sujet face à l'objet; or parmi la variété de systèmes imaginables, le langage est celui (le seul peut-être?) qui possède cette propriété remarquable de pouvoir représenter le sujet par une fonction grammaticale $^{2}$. A l'intérieur du langage, le sujet est défini d'abord d'une manière abstraite, mais il peut «penser» son rapport à l'objet (ou, plutôt, à ses différents substituts-signes). Complètement déterritorialisé, il ne peut toutefois se reconnaître autrement que comme personne grammaticale: un deuxième mouvement doit donc être introduit, niant, dans un espace déjà investi par le langage, la rupture, l'interdit initial. C'est sous une forme symbolique - et à certaines conditions il va sans dire que les retrouvailles avec l'objet du désir pourront avoir lieu, et que la possibilité de renouer avec le processus originel sera réaffirmée. Retrouvailles toujours décevantes, car elles ne le mettent jamais directement en présence de l'objet, mais seulement de ses images spéculaires. La promesse qu'elles renouvellent rend toutefois possibles les identifications successives - 1'investissement affectif objectal - et, par conséquent, permettent au sujet d'acquérir un contenu et de se constituer par une série de sédimentations dans le champ symbolique ${ }^{3}$.

Le sujet se construit donc par une succession d'identifications à l'objet du désir au travers de ses différents objets-substituts. Chaque phase le structure comme signe, au sens piercien du terme, c'est-à-dire comme une tiercéité où l'identification est le mode d'action du «Je» (ou du "Nous») sur l'objet; la séquence d'identifications successives est alors formellement homologue à la sémiosis ${ }^{4}$. Ainsi construit, le sujet n'a certes aucune parenté avec son homologue hégélien; les aliénations successives ne le mettent guère en présence de son essence objectivée, mais lui rendent plutôt accessibles des matériaux récupérés dans les

2 Dans la mesure où tout discours, peu importe son matériau sémiotique, implique un rapport du sujet à l'objet, il comporte un recours, ne serait-ce qu'imaginaire, à la langue; c'est dans ce sens que la sémiologie - en autant que l'étude des discours en fasse partie, ce qui est loin d'être unanimement admis - serait subordonnée à la linguistique, comme l'a suggéré Roland Barthes.

3 C'est la perspective adoptée par Freud dans les années vingt pour rendre compte de la structuration du Moi-Surmoi et de la constitution des groupes.

4 Peirce dira d'ailleurs que l'homme n'est rien d'autre qu'un signe engagé dans un processus de sémiosis illimitée. 
lieux balisés par l'Autre. Il ne peut guère aspirer à réaliser, par une suite de totalisations, son essence, et atteindre ainsi la Liberté.

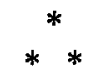

La position du sujet peut être décrite selon trois axes déterminant six relations de base. Elles se bornent à formaliser les conditions permettant de dépasser la rupture du processus élémentaire et, à ce titre, impliquent toujours un rapport déterminé à l'Autre. Le premier axe oppose le sujet démiurge au sujet «acquéreur»: le premier dénie à l'Autre la fonction de gardien de l'objet du désir et entreprend lui-même de recréer, avec les éléments à sa disposition, les conditions de production du processus originel; une telle entreprise n'est certes possible que sous une forme symbolique (la rupture de l'immédiateté, lorsqu'elle est consommée, devient irréversible). Le second reconnaît à l'Autre l'autorité découlant de son rôle et vise à entrer dans ses bonnes grâces en s'efforçant de satisfaire aux conditions édictées.

Le deuxième axe oppose la projection à l'introjection; la position du sujet est déterminée ici par le choix de la «technique» productive:

a) (identification projective) le sujet est «absorbé» par l'objet, il n'existe qu'en fonction de l'objet, perçu comme extérieur, infiniment précieux ou abject (par exemple, il se fond dans le groupe, se met inconditionnellement au service d'une idée, d'un parti, d'une religion ou d'une nation, ou, au contraire, s'y oppose, lui vouant une haine implacable);

b) (identification introjective) le sujet «absorbe» l'objet, se transformant du même coup, soit en centre narcissique de l'univers (il est en communion avec le groupe et en partage l'identité comme sa nature intime, il fusionne avec l'Univers ou avec la divinité), soit en acquérant ainsi une qualité susceptible de lui garantir l'accès à l'objet du désir.

Le troisième axe départage les dimensions symboliques et imaginaires de l'objet:

c) (symbolique) la représentation de l'objet est confondue avec l'objet lui-même et devient par conséquent son substitut adéquat, pouvant s'intégrer dans une machine désirante. L'objet devient ainsi «réel»;

d) (imaginaire) le sujet reconnaît qu'il ne s'agit guère de l'objet du désir lui-même, mais seulement d'une clef permettant éventuellement de l'atteindre, ou encore d'un simulacre. Dès lors, l'objet du désir ne peut se 
trouver dans le monde «réel» et une nouvelle dimension (l'imaginaire) doit être ouverte pour lui garantir un lieu d'existences.

Soulignons toutefois qu'il ne s'agit que d'une description de possibilités statiques, pouvant déboucher sur des itinéraires très complexes. Il n'est pas question d'entreprendre ici l'élaboration d'une typologie des variantes combinatoires autorisées par une telle description, pas plus que d'essayer de classer les sujets selon leur «contenu»: une identification projective imaginaire peut caractériser aussi bien la pratique scientifique que la théologie transcendante, le déplacement vers le symbolique étant perçu, dans un cas comme empirisme vulgaire, dans l'autre, comme glissement vers l'idolâtrie. La crainte de perdre l'objet du désir est le ressort dynamique qui motive ces déplacements et oblige le sujet à se constituer dans le champ symbolique: prenons à titre d'exemple une identification projective symbolique à un groupe social sous une forme élémentaire; le sujet est pour ainsi dire vide, n'existant que par sa soumission à ce que le groupe représente. Les qualités - la nature - du groupe sont convoitées, bien que le sujet ne puisse y accéder qu'en se faisant admettre dans son sein. Il peut arriver toutefois que cette admission soit conditionnelle: la menace de l'exclusion force alors le sujet à s'identifier introjectivement - à «absorber» - une qualité particulière du groupe, permettant de maintenir son adhésion (ce qui est souvent codifié sous forme de rituels initiatiques). Une série d'oscillations projection/introjection, s'évertuant à préserver la position initiale du sujet face à l'objet du désir - l'appartenance au groupe qui en est le dépositaire -, mais débouchent finalement sur le renversement de leur relation: le sujet s'incorpore en entier la substance du groupe, et ce dernier peut alors se disperser, voire disparaître, sans pour autant compromettre la fixation libidinale. À la limite, le sujet peut introjecter l'objet si complètement, qu'il se perçoit comme le seul digne représentant de son espèce, et les membres du groupe initial lui semblent être de vulgaires imposteurs. Dans la plupart des cas, le passage de la projection à l'introjection totale ne peut toutefois arriver à terme; les autres identifications objectales antérieures ou concurrentes, l'impossibilité d'accéder aux certains signes (de richesse, de naissance, etc.), ou le manque de «talent», peuvent être autant d'obstacles empêchant d'acquérir une «qualité» particulière. Mais cela a peu d'importance: le bénéfice de l'immersion dans le groupe réside précisément dans la possibilité de suppléer à ce manque par la participation collective à l'objet du désir; en autant que son appartenance ne soit pas menacée, le sujet

5 C'est donc presque toujours par la suite d'une déception-désillusion qu'un objet imaginaire est institué, comme le montre $O$. Manoni, dans Clefs pour l' imaginaire. 
peut s'approprier, dans l'imaginaire, la totalité des caractéristiques du groupe, même s'il sait qu'elles lui sont extérieures. C'est précisément ce qui se passe dans les groupes nationaux ou ethniques, dont chaque membre tire fierté des exploits d'un autre, pouvant ainsi réaliser par procuration les désirs autrement impossibles.

Nous constatons à quel point l'être et l'avoir ont parti lié dans le passage de l'identification projective à l'introjective: s'opposant parfois, ils tendent à se superposer ailleurs; être quelque chose et avoir quelque chose deviennent, dans certaines circonstances, presque synonymes. Ceci n'est guère étonnant lorsque l'objectivation est conçue comme substantialisation: être en mesure d'entrer dans un processus productif est lié à la possession (introjection) d'une qualité rendant le sujet apte à l'entreprendre. Mais simultanément, lorsque l'introjection et la projection reprennent leurs valeurs oppositionnelles, l'être et l'avoir vont également signifier les relations différentes, révélant du même coup la dualité de l'objet du désir. Celui-ci peut exister comme symbole de l'objet originel, et sa possession peut être convoitée directement, comme élément central des processus de production désirante. Mais il peut exister aussi comme équivalent général, donc indirectement, par sa «valeur d'échange». C'est surtout sous cette forme que l'objet est destiné à être introjecté ${ }^{6}$. Naturellement, la confusion est toujours possible entre ces deux niveaux, l'équivalent général pouvant devenir le symbole de ce qu'il devait, à l'origine, servir à racheter, et occuper ensuite la place de l'objet du désir originel.

Un autre type de déplacement se produit sur l'axe symbolique/imaginaire: ainsi, lors d'une identification projective à un objet symbolisé par le groupe, le passage du symbolique à l'imaginaire dépouille le groupe de son statut de gardien de l'objet du désir, dessinant la figure de «désillusion»; si l'identification projective se renouvelle, la répétition de cette figure trace le schéma de la quête inlassable que nous retrouvons souvent chez certains individus, cherchant par des attachements successifs aux idées et organisations les plus diverses - et parfois diamétralement opposées -, à combler un sentiment de vide existentiel. Mais d'autres trajectoires plus complexes sont possibles: une série débutant par une identification projective à un objet réel, suivie de l'introjection d'une partie de cet objet conduisant à la

6 La confusion entre ces modalités de l'objet est courante: ainsi Christianne Olivier s'est efforcée de bâtir une symétrie du développement des hommes et des femmes en utilisant comme synonymes les expressions «avoir envie de » (dans le sens de «vouloir avoir») et «envier» (dans le sens de «vouloir être»). 
«désillusion» (c'est-à-dire à l'affirmation de l'existence imaginaire de l'objet du désir, auquel le sujet continue de s'identifier projectivement), amorce une séquence développementale. L'itinéraire ainsi esquissé peut se prolonger, voire devenir sans bornes: le monde réel semblera parsemé d'objets partiels, signes-clés dont l'agglomération cumulative conduira, à l'infini, aux retrouvailles suprêmes. C'est le schéma d'une quête initiatique, du «développement» du sujet, ou encore de la recherche de la vérité scientifique qui doit couronner une démarche illimitée.

Une autre figure mérite d'être signalée: il s'agit de l'identification projective à ce que nous avons appelé «corps étranger»: ce dernier apparaît ici comme le substitut de l'objet du désir, mais d'une polarité inversée: c'est l'objet de haine - l'autre (objet) - qui, sous sa forme externalisée, est voué à la destruction. Freud décrit la fonction que cet autre joue dans la constitution de la foule, plus particulièrement des foules spontanées, à l'occasion des pogromes par exemple: sa destruction devant recevoir l'aval de l'Autre, le sujet emploi un stratagème simple: identifier à la place de l'Autre un personnage autorisant l'opération, au moyen d'un investissement libidinal.

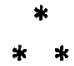

La position de l'Autre dans la production désirante est éminemment stratégique: c'est lui qui détient le pouvoir de rendre accessible l'objet du désir, c'est donc aussi lui qui fixe les conditions de sa restitution. Déterminer ses substituts et ses équivalents, dresser les plans des machines désirantes et en fixer les règles de fonctionnement, devient ainsi son attribut exclusif. A mesure que les différents personnages passent par la place de l'Autre, la production désirante glisse irrémédiablement vers le domaine social. Quels acteurs sont susceptibles d'occuper cette position, comment y accèdent-ils et de quelle manière s'acquittentils de leur tâche? Questions capitales, mais auxquelles il est impossible de répondre ici. Nous ne les aborderons que par un aspect, celui où l'Autre se fait nomothete, ${ }^{7}$ et fixe les contours des objets offerts ensuite au désir; situation conférant un pouvoir considérable, mais qui est loin d'être de tout repos: il faut constamment réconforter l'identification à l'Autre dont dérive la légitimité du rôle; mais surtout, il faut assumer les dangers découlant des ambiguittés de la position même, rappelés avec force dans le mythe freudien du meurtre du père.

$7 \mathrm{Ill}$ s'agit, bien sûr, d'une fonction sociale (et non d'un personnage particulier) pouvant être assumée par des acteurs divers. 
Tracer les frontières a été de tout temps l'oeuvre sacrée par excellence; les traverser relevait tantôt du jeu contestataire, tantôt d'une démarche initiatique confirmant leur efficacité symbolique. Mais tracer une frontière, c'est avant tout ouvrir une discontinuité, procéder à un marquage: il faut fonder la topographie d'un espace imaginaire à l'aide de signes plus ou moins lisibles, et dresser une carte où l'intérieur et l'extérieur deviennent lisibles. Pour rendre ce tracé efficient, le nomothète doit se mettre à la place de l'Autre: il ne s'agit pas seulement de procéder à un classement, mais aussi d'en garantir la légitimité, c'est-à-dire sa valeur productive dans l'économie désirante. Chaque opération met ainsi à l'épreuve la solidité des rapports symboliques qui circonscrivent le pouvoir d'énoncer la parole vraie (c'est-à-dire pouvant être investie par le désir).

Parmi les systèmes de classement, celui du nomothète possède une caractéristique particulière: il vise à arracher au chaos un espace clos et ordonné, à séparer le pur de l'impur, et à exorciser le démoniaque en le repoussant au-delà du cercle sacré. Il crée les figures de l'autre, de l'étranger, du déviant, de l'informe.

L'action du nomothète peut revêtir deux formes de base: d'abord celle d'une intervention magique, c'est-à-dire découlant du pouvoir de la parole vraie sur le réel. Il s'agit alors d'un geste proprement démiurgique - celui du sorcier aussi bien que d'une Assemblée législative. En tant que magicien, le nomothète occupe pleinement la place de l'Autre et maîtrise directement les forces du monde: il est le créateur, serait-ce ex nihilo, de toute chose. La deuxième forme, par contre, met en oeuvre non un pouvoir, mais un savoir spécial: le nomothète n'est plus une incarnation de l'Autre, mais son représentant et interprète. Position plus prudente, et tout aussi efficace: 1'affirmation insolente et dominatrice, face au sujet et à l'univers, fait place à une lecture patiente de l'ordre du monde, au déchiffrement des signes dont une puissance souveraine autonome (des dieux, de la Nature, de la Société,... bref, l'Autre véritable) aurait balisé son oeuvre. En tant qu'exégète, le nomothète n'institue pas la frontière entre le bien et le mal, il la découvre inscrite déjà là, dans la texture du réel. Aussi son autorité apparaît d'emblée comme rationnelle, dérivée de sa capacité éminente d'interpréter la parole fondatrice de l'Autre.

Si les divisions du monde ne sont plus immédiatement lisibles, si la reconnaissance de la nature et des limites des choses nécessite la médiation du savoir, le pouvoir de l'exégète est conforté encore 
davantage par l'incertitude du profane quant à sa position dans le champ symbolique. Le langage profond et originel, dont l'exégète est seul à posséder la clé, doit donc se doubler d'un système de signes manifestes, balisant les itinéraires permis et marquant les frontières des espaces interdits. Un travail important de fabrication d'emblèmes et de recherche de stigmates accompagne donc nécessairement l'oeuvre du classement social. En prenant le résultat de cet effort pour l'objet d'investissement affectif, les individus deviennent membres d'un groupe et se constituent en sujet social.

Dans la constitution du groupe, tout se passe comme si à l'interdit originel de l'inceste, s'était substitué l'obligation de l'inceste symbolique, une contrainte endogamique faisant office d'opérateur de classement social. $\mathrm{La}$ destruction des processus élémentaires brise la circularité de la vie immédiate (disons, la symbiose mère-enfant), forçant le sujet à s'aventurer dans les espaces illimités de l'altérité; cette tâche accomplie, il faut établir de nouvelles territorialités, se repérer (acquérir une nature, une identité) dans les espaces symboliques (disons, atteindre à de nouvelles symbioses individu-groupe). D'un côté, l'ouverture sur un ailleurs, de l'autre, le repli sur le déjà-là. La structure logique de cette métaphore traduit donc le passage de l'unité indifférenciée à l'unité comme absence des différences.

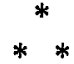

Les positions occupées par le sujet face à l'objet du désir peuvent être expérimentées, directement ou d'une manière ludique, dans les performances discursives. Le discours, tel que nous allons l'envisager ici, ne sera pas une «sémiologie» au sens de Hjemslev, mais plutôt une machine productive, dont les éléments constitutifs sont de nature sémiotique; ne contenant pas de signification donnée d'avance, il est destiné à en produire. Qui plus est, ce n'est qu'un moyen pour obtenir des effets de différents ordres (esthétiques, magiques, représentatifs,...). En tant que machine, le discours ne peut toutefois fonctionner que branché sur un sujet, qui est la source énergétique susceptible de mettre en mouvement ses éléments. Cette dépendance rend chaque oeuvre discursive vulnérable aux assauts du temps, et subordonne son emploi à l'identité sociale de ses consommateurs: comme par magie, une mutation dans la texture de la société peut enlever toute efficacité aux formes discursives jadis puissantes, voire rendre tout à fait impossible même leur existence. 
Nous définirons le discours par deux dimensions constitutives: un texte et un Code $^{8}$. Le Code est spécifié, d'une manière générale, par un ensemble hiérarchisé de signes, distribués aussi bien à l'intérieur du texte qu'à sa périphérie, les éléments contextuels pouvant parfois prendre une importance décisive. Il ne fournit pas la clé d'une signification, mais précise les règles de sa production. Il s'ensuit que le même texte, muni de deux codes différents, peut produire des effets et des sens très divers: des expériences simples (disposer un article journalistiques purement informatif sous la forme de vers libres; remplacer, dans certaines oeuvres, l'indication «roman» par celle de «biographie», etc.) nous en convaincraient aisément. L'étude du discours ne relève donc pas d'une «sémiotique générale», mais d'une théorie de la production (de la signification et des effets).

Il nous suffira de cerner ici un aspect du Code, mais de loin le plus fondamental, qui réside dans l'induction d'un rapport du sujet à l'objet du discours; par là nous pouvons élaborer une première typologie, certes très générale, mais utile pour l'intelligence de notre problématique. Ce rapport spécifie, du moins en partie, la relation retenue entre l'objet du discours et l'objet du désir. Quelques exemples à peine pourront illustrer cette question.

Le premier critère oppose deux types de discours: l'efficacité du premier nécessite que le sujet s'identifie à son producteur, celle du second, que le sujet soit leur utilisateur. Tel est le cas du discours magique par exemple, ne pouvant avoir prise sur le réel que si le Code place le sujet en position de producteur (en fait, de l'Autre). Mais il en est ainsi également dans le discours savant, dont l'efficacité - capacité de rendre intelligible le réel - implique que le sujet refasse, sur les traces de l'auteur, le chemin de la connaissance. Dans les deux cas, le discours est un instrument productif que le sujet emploie - parfois avec le consentement, voire la complicité de l'Autre, parfois en affirmant sa révolte ou son autonomie - pour atteindre l'objet du désir.

Un deuxième critère concerne le «degré de liberté» de l'objet du discours (grosso modo, son degré d'indétermination par une «réalité» extradiscursive). Ceci se traduit, en premier lieu, par la nature particulière de l'épreuve de commutation applicable: dans le discours

8 La majuscule sert à distinguer le sens que nous donnons ici au terme de celui, plus courant, d'un ensemble de règles mettant en forme le plan de représentation et celui de contenu, tout en explicitant leurs correspondances. Le «texte» doit être entendu au sens utilisé par Umberto Eco, dans La structure absente. 
savant ou magique, cette liberté est à peu près nulle. L'objet du désir détermine l'objet du discours et toutes les variations du plan de l'expression, conservant le même objet, doivent être tenues pour équivalentes (ce qui signifie qu'elle ne sont pas pertinentes au point de vue de la production du sens). Il en est ainsi également dans le discours nomothétique (un livre sacré, par exemple), dans lequel l'objet du discours est identifié à l'objet du désir; l'exégèse - le rapport de production à l'égard du discours - force alors le sujet à se déplacer jusqu'à parvenir à cette identification. Il en va tout autrement dans le discours fictif: affirmer le caractère imaginaire de son objet implique son autonomie relativement à toute détermination extradiscursive. Dès lors, différentes variantes de la construction de l'objet s'avèrent possibles, et le choix d'une variante particulière devient porteur du sens (dire, dans un article journalistique, que «X a tourné à gauche», n'a de valeur qu'informative, puisque « $\mathrm{X}$ a tourné à droite» concerne une autre réalité, possible, mais qui ne s'est pas actualisée; dans un roman, par contre, tous les déplacements imaginables de $\mathrm{X}$ font partie d'une chaîne paradigmatique équiprobable; en choisir un, correspond à l'exercice d'une liberté autorisée par le Code, ce qui est nécessairement producteur de sens). Le discours fictif apparaît ainsi entièrement intentionnel; comme 1'a souligné Roland Barthes, c'est une machine qui transforme le hasard en nécessité (nous pouvons dire, à propos de l'article journalistique, que c'était «par hasard» si $X$ a tourné à gauche; dans un roman c'est impossible). L' «intentionnalité» dont il est question ici n'est, bien entendu, aucunement psychologique (celle d'un hypothétique auteur) mais bien un effet discursif. La fiction est ainsi soustraite au «principe de la réalité» et fonctionne sous l'empire du «principe du plaisir». L'indétermination est portée à son plus haut niveau dans le discours poétique, lorsque, par une sorte de négation de la négation, l'objet du discours est encore une fois identifié à un objet réel, mais cette fois-ci au discours lui-même. Par analogie avec la théorie de l'information, cette progression dans le nombre de «degrés de liberté» de l'objet peut être assimilée à la croissance de l'entropie de la source du message: dans le discours magique, la subordination stricte de l'objet du discours à l'objet du désir n'autorise qu'un seul «message»; comparativement au discours «informatif», le discours de fiction est fortement «entropique» et, à l'autre extrême, le discours poétique est hyperentropique.

Dans la mesure où le mode de fonctionnement du discours est subordonné au maintien d'un rapport particulier du sujet à l'objet du discours, chaque type discursif admet une catégorie de "vraisemblable», qui en définit la stabilité. Les fonctions productives du Code doivent 
constamment maintenir le sujet dans la position idoine, sans quoi le discours se désagrégerait (deviendrait «invraisemblable»). Mais le Code est, lui aussi, un ensemble de signes, et, à ce titre, son utilisation peut être non seulement productive, mais aussi purement signifiante. Des simulacres de discours peuvent ainsi être fabriqués, dans lesquels les indications codées ont perdu leur capacité productive de sens, et se bornent à signifier la présence d'une discursivité particulière, la subordonnant le plus souvent à une tout autre logique. Le simulacre du discours scientifique est souvent employé dans le discours fictif, mais on peut l'utiliser également dans le discours nomothétique (celui d'un politicien, par exemple, où il ne fonctionne pas, il va de soi, comme discours scientifique, mais comme garant de la légitimité du nomothète).

L'analyse du discours constitue un terrain expérimental fournissant des résultats positifs sur la constitution du sujet: c'est le discours nomothétique qui dévoile, le plus souvent, la forme de l'objet à être approprié, pour fournir au sujet les matériaux de sa construction. Dans le discours fictif, tous les mécanismes identificatoires sont en oeuvre, mettant en scène d'une manière ludique la sémiosis conduisant à l'émergence du Moi. Tout rapport à l'objet du discours se noue ainsi dans la duplicité du rapport à l'objet du désir, rendant l'efficacité discursive tributaire de la production désirante.

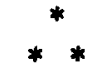

Les possibilités productives des «métissages» doivent donc être comprises dans la perspective plus large des transformations du rapport à l'objet du désir, traduisant la crainte de sa perte. En effet, chaque position du sujet comporte une menace de déstabilisation: dans une identification introjective, lorsque le sujet croit entrer en possession de la qualité convoitée, le métissage apparaît comme angoisse de la contaminer, voire d'ingérer par mégarde une «mauvaise» qualité, rendant à jamais impossibles les retrouvailles amoureuses. L'intensité des exigences de l'Autre et l'incertitude quant à la capacité de s'y conformer, se solidifient dans la haine de l'autre. Mais si la position du sujet est confortée, son identification projective peut à son tour être menacée: tout objet n'est qu'un symbole de l'objet du désir et, par conséquent, potentiellement décevant. L'attachement excessif peut se transformer ainsi en obstacle à la reconquête du grand Objet imaginaire, et le «métissage» se mue en technique créatrice, la révolte contre l'Autre amorçant la rupture d'avec le simulacre. Traverser les frontières, c'est contester le statut de nomothète à celui qui les a tracées; c'est aussi une tentative 
d'usurper soi-même la place de l'Autre, et de retrouver, ne serait que momentanément, l'autonomie du sujet dans la production désirante.

La métamorphose de l'objet en substance est l'un des effets propres au discours nomothétique; aussi les «métissages» ne peuvent avoir lieux que dans 1'univers des objets du désir. Les discours ne peuvent guère être métissés; leur mode de fonctionnement étant déterminé par la position respective du sujet à l'objet du discours, la seule confusion peut résulter d'une insuffisance (ou perversion) du Code, créant une situation d'incertitude quant à leur position. Il est vrai cependant, que dans le domaine discursif, il existe aussi des objets fabriqués par les nomothètes (Académies, aréopages critiques, Musées, et autres), et offerts en pâture au désir: la quête des «vrais» concepts - du roman, du savoir, de la poésie - ne propose le plus souvent qu'un idéal vite fétichisé, dont une nouvelle génération de «créateurs» s'empressera allégrement de violer les frontières. 